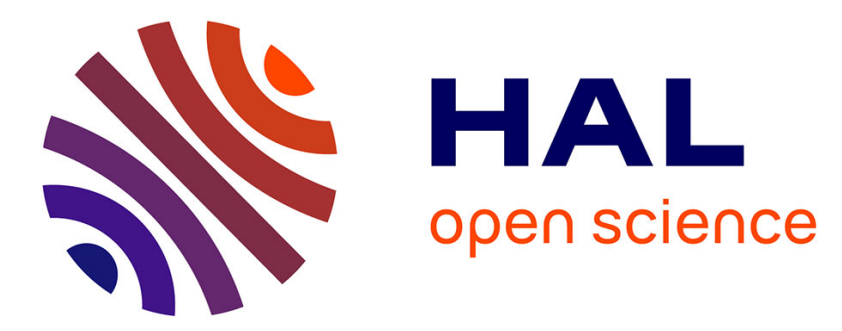

\title{
Network embedding for brain connectivity
}

\author{
Lucrezia Carboni, Sophie Achard, Michel Dojat
}

\section{To cite this version:}

Lucrezia Carboni, Sophie Achard, Michel Dojat. Network embedding for brain connectivity. ISBI 2021 - International Symposium on Biomedical Imaging, Apr 2021, Nice / Virtual, France. pp.1-4. hal-03273946

\section{HAL Id: hal-03273946 \\ https://hal.science/hal-03273946}

Submitted on 29 Jun 2021

HAL is a multi-disciplinary open access archive for the deposit and dissemination of scientific research documents, whether they are published or not. The documents may come from teaching and research institutions in France or abroad, or from public or private research centers.
L'archive ouverte pluridisciplinaire HAL, est destinée au dépôt et à la diffusion de documents scientifiques de niveau recherche, publiés ou non, émanant des établissements d'enseignement et de recherche français ou étrangers, des laboratoires publics ou privés. 


\title{
NETWORK EMBEDDING FOR BRAIN CONNECTIVITY
}

\author{
Lucrezia Carboni $^{1,2} \quad$ Sophie Achard $\quad$ Michel Dojat $^{2}$ \\ ${ }^{1}$ Univ. Grenoble Alpes, CNRS, Inria, Grenoble INP, LJK, 38000 Grenoble, France \\ ${ }^{2}$ Univ. Grenoble Alpes, Inserm, U1216, Grenoble Institut Neurosciences, GIN, 38000 Grenoble, France
}

\begin{abstract}
In Neurosciences, networks are currently used for representing the brain connections system with the purpose of determining the specific characteristics of the brain itself. However, discriminating between a healthy human brain network and a pathological one using common network descriptors could be misleading. For this reason, we explored network embedding techniques with the purpose of brain connectivity networks comparison. We proposed first the definition of representative graph for healthy brain connectivity. Then, two classification procedures through embedding are introduced, achieving good accuracy results in different datasets. Moreover, the intriguing power of this technique is given by the possibility of visualizing networks in a low-dimensional space, facilitating the interpretation of the differences between networks under diverse conditions e.g. normal or pathological.
\end{abstract}

Index Terms - Network classification, MRI, low dimensional space, graph comparison.

\section{INTRODUCTION}

In various contexts, network embedding technique has been developed for performing analysis on a single graph. This technique has been proven to work for different applications, such as node classification or link prediction. An efficient network embedding algorithm is capable of capturing the relevant features of the graph and reproduce them in a lowdimensional Euclidean space.

In the context of Neurosciences, networks are used to represent the set of connections between brain regions with eventually the aim of distinguish pathological versus healthy states. However, such a distinction could be missed based on the network descriptors commonly used. Indeed there is no clear evidence of a best measure to be used in the discrimination of different brain states [1]. As a results each process focuses on specific descriptors which differ from pathology to pathology or requires hand-crafted index to reveal a dissimilarity (i.e. [2]). In this study, we investigate the use of network embedding with the goal of applying to human brain connectivity network classification. Moreover, the intriguing power of the embedding in our domain is given by the possibility to visualize the connectivity network in a low-dimensional space, which allows to determine which brain regions and properties are peculiar of the given network and are fundamentals for the normal vs abnormal classification process, providing interestingly new knowledge about the pathological conditions.

\section{MATERIAL AND METHODS}

\subsection{Data: brain connectivity networks definition}

The definition of brain connectivity networks was achieved through out different phases proposed in [3]. First, the functional MR images (fMRI) acquired at rest were aggregated over 90 regions defined according to the anatomical labeling in [4]. For each parcel, a unique time series signal was determined by averaging the fMRI time series over all voxels of the parcel, weighted by the proportion of gray matter in each voxel, obtained by individual structural image tissue segmentation, to take into account for the partial volume effect. The following stage consisted in the application of the discrete wavelet transform to each mean fMRI time series. As a result, for each subject, different fMRI time series at distinct scales, associated to different intervals of frequencies, were at our disposal. Since it has been observed that the resting state information activity is mainly captured at a frequency lower than $0.1 \mathrm{~Hz}$, the correlation among regions was estimated only for this frequency band. Finally, by thresholding the correlation matrix, a binary matrix was defined. This matrix corresponded to the adjacency matrix of the brain connectivity network, whose nodes were given by the parcels. The threshold was tuned for each subject in order to compute a binary matrix with a given non-zeros entries number; in our experiments it has been set to 400 . Table 1 indicates the resting state datasets we used in our experiments extracted from different databases.

\begin{tabular}{cccc}
\hline Dataset name & \multicolumn{3}{c}{ Number of Data } \\
& Total & Class 1 & Class 2 \\
\hline HCP test retest [5] & $100 \times 2$ & - & - \\
Coma [2] & 37 & 20 Control & 17 Comatose \\
Young\&Elderly [6] & 26 & 15 Young & 11 Elderly \\
\hline
\end{tabular}

Table 1. List of the databases used including different classes of subjects. HCP: Human Connectome Project. 


\subsection{Nodal embedding}

Network embedding can be seen as a dimensionality reduction tool which maps a network into a vector space. We focussed on nodal embedding, namely a mapping function which maps a graph into a bag-of-vector where each vector is associated to a single vertex of the graph.

Among the available methods, we selected node2vec embedding algorithm [7] which has been proven of being able to capture the structural equivalence of nodes. node2vec is based on the Skip-gram architecture which is used to learn a features representation of words based on their context [8]. In the case of network, the concept of context is translated into the one of neighborhood. Precisely, node2 vec defines a flexible notion of a node's neighborhood depending on specific characteristics we are interested in, such as those obtained by structural relations or by similar relationships between the neighborhoods. Note that neighborhood is not defined based on a unique similarity function, but can be defined via two searching strategy. For each vertex, the algorithm computes a neighborhood set of a given number of nodes. The breadth-first sampling (BFS) considers the neighborhood of a node as nodes which are immediate neighbors of the source. Whereas, the DFS neighborhood is composed by nodes which are sequentially sampled at increasing distances from the node itself. node $2 \mathrm{vec}$ allows to smoothly interpolate between BFS and DFS.

In synthesis, the node $2 \mathrm{vec}$ embedding function is determined by the following parameters: $d$ dimension of the embedding space, $N$ number of random walks per node used to estimate the proximity matrix, $L$ random walk length, $k$ size of the neighborhood set for each node, $p$ return parameter and $q$ in-out parameter controlling the sampling strategy (small $p \rightarrow$ BFS, small $q \rightarrow$ DFS).

Setting the parameters' values referring to a single graph's embedding would result in the necessity of using different parameters for each single graph. However, in this way we loose the possibility of a fair graphs comparison. Because our purpose is to capture the class features for different graphs, we propose to set these parameters according to the results obtained for a graph comparison for which we know the ground truth. To clarify, we would like to perform a graph embedding for which the computed similarity index for the same class is maximized, while the similarity index between different classes is minimized. The obtained embedding, thereby, would eventually be able to capture the shared characteristic of a group of graphs. Since all the graphs in our datasets are comparable in terms of the number of nodes and edges, we chose to tune the parameters based on the HCP dataset. This dataset provides test and retest data for the same subjects. Thus, using this unique dataset for the tuning, we expected to maximize the similarity index between graphs of the same subject, forcing the embedding to capture the characteristics which were relevant in brain connectivity. Indeed, a parameters configuration which estimates similar embedding for similar graphs, was assumed to capture the intrinsic nature of the corresponding brain connectivity network.

The final parameters' values were $d=3, N=20, L=$ $2 \times$ network diameter, $k=3, p=1, q=2$.

\subsection{Pyramid graph matching kernel}

In our project, we are interested in comparing networks which belong to different classes, healthy vs pathological subjects or for patients sub-typing. This comparison could be directly performed in the network space; however, our purpose was to explore the potentiality of embedding in the analysis of brain connectivity. With this goal, we found the work [9] newsworthy. In particular, inspired by the pyramid match kernel used in computer vision, the authors have designed an equivalent version for graphs, named pyramid matching graph kernel. The main idea of the algorithm was to count the matching of vectors in the embedding space at different resolution levels. We reproduced their setting using node $2 \mathrm{vec}$ as embedding algorithm. In the embedded space, given a number of level, a grid of cells having increasing size in one dimension for each level was computed. Two vectors corresponding to the same region in the brain were matched if they belonged to the same cell. Each matching was weighted according to the dimension of the corresponding region. Counting the number of matchings between two embedded graphs allowed to compute their similarity.

\subsection{Representative graph}

Previous works have defined, for a class, an average graph in the network space, taking into account the average of the adjacency matrices or selecting edges present in all the graphs of the class. We propose an innovative approach using a network embedding algorithm. It requires labeled graphs with labeled nodes. Note that all graphs have the same number of nodes. Having at our disposal a labeled set of graphs, we considered all the networks which belong to the same class. For each node, we considered the set of the embedding vectors obtained through the mapping of all graphs of the class. Then, we averaged components by components and computed the barycenter. The output of this process was a set of vectors, where each vector was associated to a given node in the graph. This output can be interpreted as a virtual embedding of a representative graph. In fact, we were implicitly assuming that the vector position in the embedding space should be related to the label associated to the node. Moreover, since the embedding was supposed to capture the structural equivalence of nodes, we hypothesized that for individuals belonging to the same class, same brain regions exhibited the same connectivity pattern.

\subsection{Classification procedure}

We propose to compare two classification procedures through embedding, standard SVM $v s$ a graph representation of a class 


\begin{tabular}{|c|cc|cc|}
\hline & C1 - MA & C1 - SD & C2 - MA & C2 - SD \\
\hline COMA & 0.83 & 0.019 & 0.75 & 0.025 \\
\hline Y\&E & 0.59 & 0.045 & 0.62 & 0.051 \\
\hline
\end{tabular}

Table 2. Classification performances over all the datasets through the two different classification procedures. MA mean accuracy and SD - standard deviation computed on 100 experiments.

(see Fig.1). For both procedures, we computed the embedding of all networks using node2vec. As a result, each graph was converted into a set of vectors. We randomly determined a subset of the dataset as the training set. The remaining data formed the candidates for classification (testing set). The training set was partitioned into two classes.

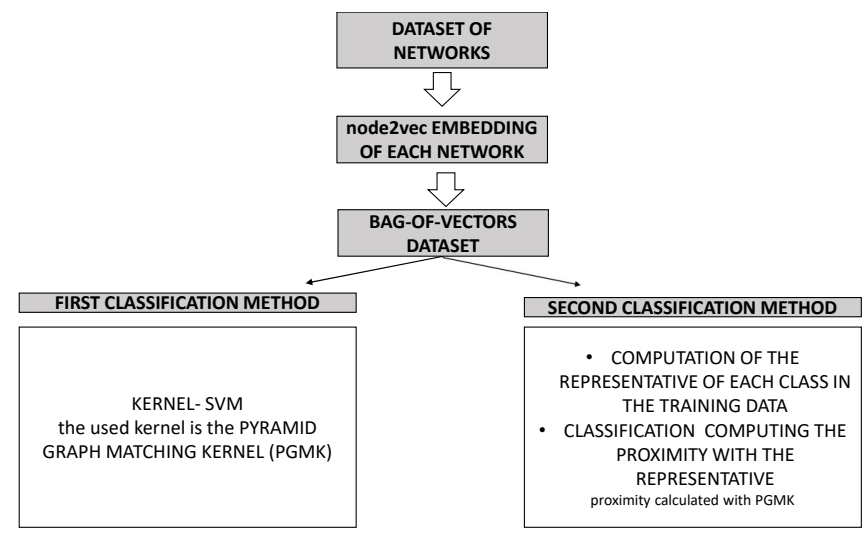

Fig. 1. Classification procedure scheme

\subsubsection{First classification procedure: $\mathrm{Cl}$}

We calculated the gram matrix using the pyramid graph matching kernel. Then, we used kernel-SVM to predict the labels on the candidates in the testing set. The kernel used was the pyramid graph matching kernel.

\subsubsection{Second classification procedure: $C 2$}

This procedure used the average graph as a representative for each class for the comparison. The average embedding graph was computed for each class. To classify the testing set, we computed the pyramid graph matching similarity index between each candidate and the two graph representatives of the classes. We predicted the graph label, according to the more similar graph.

\section{EXPERIMENTS AND RESULTS}

First, we compared the representative networks obtained from the different groups of controls coming from the different datasets. The first three graphics in Fig. 2 is illustrating the robustness of the construction of the representative graphs obtained from the three different datasets acquired in different conditions, but preprocessed using the same methods. Second, we ran the two classification procedures 100 times for the datasets. Each time the embedding and the training sets were computed in a repeated random sub-sampling validation framework. We observed a slightly variability in terms of accuracy depending on the datasets.

\section{DISCUSSION}

Network representation is largely used in Neurosciences for structural or functional brain connectivity studies. In this paper we explored the applicability of the network embedding approach for brain connectivity investigation. We calibrated the embedding method parameters based on one dataset considered as the ground truth. It is important to mention that this was not done to improve performances but to favor a certain pattern on the data. Besides, the dataset used for calibration was indeed very different in term of acquisition conditions (different centers, different MR scanners) that the other datasets we considered then for classification performances testing.

Our preliminary results demonstrate, using datasets coming from different databases, that a network embedding approach, combined with standard classification methods, provides good performances for separating two different families of graphs representative of pathological states (brain trauma, coma) versus healthy conditions or normal aging connectivity changes (young vs elderly). The performances were very similar for all the datasets, representative of very different clinical situations, with the classification method that used a graph representative of a class of subjects versus the more standard SVM method. This demonstrates that our definition of the graph representative of a class was valid. However, we notice that SVM provided the best performances for the coma dataset being able of capturing the dissimilarity between the two classes without any prior selection of graph descriptors neither requiring the definition of a specific measure [2]. The performances remained appreciable for Young\&Elderly.

Our classification scores are good, but not excellent. However, we consider that our procedure should not be only evaluated on its classification results. Indeed, the major interest of network embedding is to facilitate the interpretation of the differences in a low-dimensional space between networks (see Fig. 2). This is highly valuable in Neurosciences where networks may be representative of brain connectivity under different conditions e.g. normal or abnormal. Thus, even whether previous works may report better classification performances, we assume that a network embedding procedure provides an additional value: the possibility of determining a signature for the class.

The use of global descriptors for graph characterization has been proven not to be enough for detecting some differences between graphs [2]. Our achievement shows the power of our embedding method for graphs comparison in capturing the meaningful features of the network. Even if further analysis 


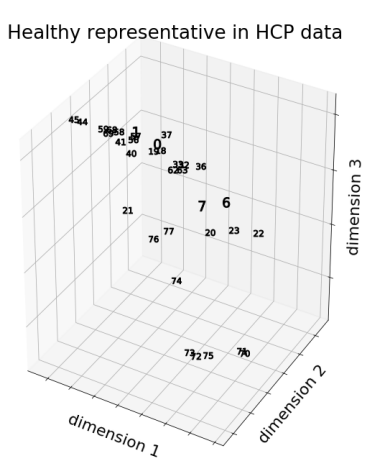

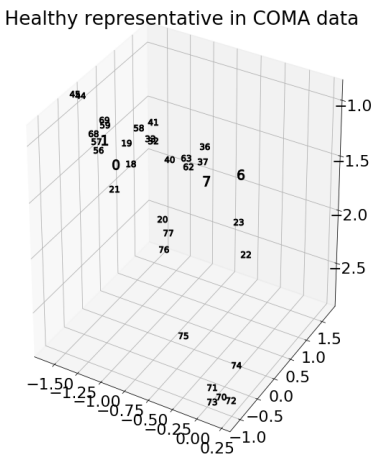

(a)
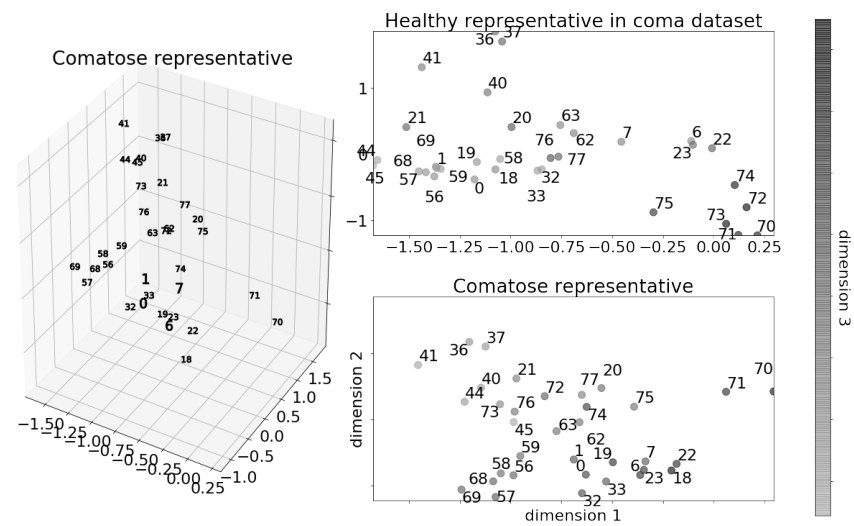

(b)

Fig. 2. (a) Visualization in a 3D space of a subset of regions in different representative embeddings. Qualitatively, we can appreciate the closeness of the healthy representatives extracted from different datasets. While, major differences could be visualized with respect to the right picture representing the comatose class. Numbers refer to the region labels in [4].

(b) Projection over the first two dimensions of the embedded vectors in the healthy control (top) and comatose (bottom) representatives in COMA data. Points' marker are colored according to the values of the third coordinate.

needs to be conducted, we interpret the good performances obtained as an indicator of the type of graph property preserved in the embedding.

\section{COMPLIANCE WITH ETHICAL STANDARDS}

This research study was conducted retrospectively using human subject data made available by the HCP open source. Ethical approval was not required as confirmed by the license attached with the open data. The use of the other datasets was approved by the ethical committee consulted for each study and all the concerned participants or next of skin signed the informed consent form.

\section{ACKNOWLEDGEMENTS}

LC was supported by MIAI@Grenoble Alpes (ANR 19P3IA-003). The authors have no relevant financial or nonfinancial interests to disclose.

\section{REFERENCES}

[1] Michael N Hallquist and Frank G Hillary, "Graph theory approaches to functional network organization in brain disorders: A critique for a brave new small-world," Network Neuroscience, vol. 3, no. 1, pp. 1-26, 2018.

[2] Sophie Achard, Chantal Delon-Martin, Petra E Vértes, Félix Renard, Maleka Schenck, Francis Schneider, Christian Heinrich, Stéphane Kremer, and Edward T Bullmore, "Hubs of brain functional networks are radically reorganized in comatose patients," Proceedings of the National Academy of Sciences, vol. 109, no. 50, pp. 20608-20613, 2012.

[3] Sophie Achard, Raymond Salvador, Brandon Whitcher, John Suckling, and ED Bullmore, "A resilient, low- frequency, small-world human brain functional network with highly connected association cortical hubs," Journal of Neuroscience, vol. 26, no. 1, pp. 63-72, 2006.

[4] Nathalie Tzourio-Mazoyer, Brigitte Landeau, Dimitri Papathanassiou, Fabrice Crivello, Olivier Etard, Nicolas Delcroix, Bernard Mazoyer, and Marc Joliot, "Automated anatomical labeling of activations in spm using a macroscopic anatomical parcellation of the mni mri single-subject brain," Neuroimage, vol. 15, no. 1, pp. 273-289, 2002.

[5] Maité Termenon, Assia Jaillard, Chantal Delon-Martin, and Sophie Achard, "Reliability of graph analysis of resting state fmri using test-retest dataset from the human connectome project," Neuroimage, vol. 142, pp. 172187, 2016.

[6] Sophie Achard and Ed Bullmore, "Efficiency and cost of economical brain functional networks," PLoS Comput Biol, vol. 3, no. 2, pp. e17, 2007.

[7] Aditya Grover and Jure Leskovec, "node2vec: Scalable feature learning for networks," in Proceedings of the 22nd ACM SIGKDD international conference on Knowledge discovery and data mining, 2016, pp. 855-864.

[8] Tomas Mikolov, Kai Chen, Greg Corrado, and Jeffrey Dean, "Efficient estimation of word representations in vector space," arXiv preprint arXiv:1301.3781, 2013.

[9] Giannis Nikolentzos, Polykarpos Meladianos, and Michalis Vazirgiannis, "Matching node embeddings for graph similarity," in Thirty-First AAAI Conference on Artificial Intelligence, 2017. 